\title{
Carbonyl-Coordinating Polymers for High-Voltage Solid-State Lithium Batteries: Solid Polymer Electrolytes - ERRATUM
}

doi: https://doi.org/10.1557/mre.2020.3

This article [1] was published in the incorrect volume and has since been corrected. The publisher apologizes for the error.
Hongli Xu, Jingbing Xie, Zhongbo Liu, Jun Wang, and

Yonghong Deng

REFERENCE:

1. Xu H., Xie J., Liu Z., Wang J., and Deng Y. (2020). Carbonyl-Coordinating Polymers for High-Voltage Solid-State Lithium Batteries: Solid Polymer Electrolytes. MRS Energy \& Sustainability, 7, E2. https://doi.org/ $10.1557 / \mathrm{mre} .2020 .3$ 